\title{
Six Metabolism Related mRNAs Predict the Prognosis of Patients With Hepatocellular Carcinoma
}

\section{OPEN ACCESS}

Edited by: Miroslaw Kornek, Universität Bonn, Germany

Reviewed by: Tathiane Malta University of São Paulo, Brazl Artur Slomka,

Nicolaus Copernicus University in Toruń, Poland

Tudor Mocan

I luliu Hațieganu University of Medicine and Pharmacy, Romania

*Correspondence: Baogang Peng pengbg@mail.sysu.edu.cn Wei Chen

chenw57@mail.sysu.edu.cn

${ }^{+}$These authors have contributed equally to this work

Specialty section: This article was submitted to Molecular Diagnostics and Therapeutics,

a section of the journal Frontiers in Molecular Biosciences

Received: 25 October 2020 Accepted: 11 January 2021 Published: 25 February 2021

Citation: Wu X, Lan T, Li M, Liu J, WuX, Shen S,

Chen $W$ and Peng $B$ (2021) Six Metabolism Related mRNAs Predict the Prognosis of Patients With

Hepatocellular Carcinoma. Front. Mol. Biosci. 8:621232. doi: 10.3389/fmolb.2021.621232
Xiwen $W u^{1 \dagger}$, Tian Lan ${ }^{2 \dagger}$, Muqi $L^{1}{ }^{1}$, Junfeng Liu ${ }^{3}$, Xukun $W u^{1}$, Shunli Shen ${ }^{1}$, Wei Chen ${ }^{2 *}$ and Baogang Peng ${ }^{1 *}$

${ }^{1}$ Department of Hepatic Surgery, The First Affiliated Hospital of Sun Yat-sen University, Guangzhou, China, ${ }^{2}$ Department of Pancreaticobiliary Surgery, The First Affiliated Hospital of Sun Yat-sen University, Guangzhou, China, ${ }^{3}$ Department of Pathology, The First Affiliated Hospital of Sun Yat-sen University, Guangzhou, China

Background: Hepatocellular carcinoma (HCC) is one of the most common aggressive solid malignant tumors and current research regards $\mathrm{HCC}$ as a type of metabolic disease. This study aims to establish a metabolism-related mRNA signature model for risk assessment and prognosis prediction in HCC patients.

Methods: HCC data were obtained from The Cancer Genome Atlas (TCGA), International Cancer Genome Consortium (ICGC) and Gene Enrichment Analysis (GSEA) website. Least absolute shrinkage and selection operator (LASSO) was used to screen out the candidate mRNAs and calculate the risk coefficient to establish the prognosis model. A high-risk group and low-risk group were separated for further study depending on their median risk score. The reliability of the prediction was evaluated in the validation cohort and the whole cohort.

Results: A total of 548 differential mRNAs were identified from HCC samples $(n=374)$ and normal controls $(n=50)$, 45 of which were correlated with prognosis. A total of 373 samples met the screening criteria and there were randomly divided into the training cohort $(n=186)$ and the validation cohort $(n=187)$. In the training cohort, six metabolism-related mRNAs were used to construct a prognostic model with a LASSO regression model. Based on the risk model, the overall survival rate of the high-risk cohort was significantly lower than that of the low-risk cohort. The results of a time-ROC curve proved that the risk score $(A \cup C=0.849$ ) had a higher prognostic value than the pathological grade, clinical stage, age or gender.

Conclusion: The model constructed by the six metabolism-related mRNAs has a significant value for survival prediction and can be applied to guide the evaluation of HCC and the designation of clinical therapy.

Keywords: metabolism, hepatocellular carcinoma, the cancer genome Atlas, mRNAs, prognostic model 


\section{INTRODUCTION}

Hepatocellular carcinoma (HCC) is one of the most aggressive solid malignant tumors with poor prognosis and high mortality. An article on global cancer statistics for 2018 reported that HCC was the third leading cause of cancer-related deaths worldwide (Bray et al., 2018). Only a small number of HCC patients were able to receive curative treatment including hepatectomy resection, transplantation, or ablation at the time of diagnosis. What's more, even after curative therapy, tumor recurrence occurs in $50-70 \%$ of HCC patients within 5 years (Forner et al., 2012). Most of the studies focus on the clinicopathological characteristics of HCC patients to construct prognostic models to predict overall survival (OS) and help to stratify patients' OS (Xu et al., 2018; Best et al., 2020; Dai et al., 2020).

Recent years have seen a gradual increase in the number of reports on the use of genomics, proteomics and metabolomics technology to study the pathogenesis and tumorigenesis of malignant tumors (Kimhofer et al., 2015; De Stefano et al., 2018; Beyoglu and Idle, 2020). Since metabolic disorder is a key event in the occurrence and development of HCC, HCC has been considered a type of metabolic disease (Piccinin et al., 2019). An increasing number of characteristic metabolic markers related to HCC have been found. Examples include alpha-fetoprotein (AFP) (Yamamoto et al., 2010; Notarpaolo et al., 2017; Pinyol et al., 2019) and PIVKA-II also called des$\gamma$-carboxyprothrombin (DCP) (De Stefano et al., 2018; Loglio et al., 2020). These markers can not only indicate the natural course of a tumor, but also provide evidence for a treatment strategy and an evaluation of the pharmacodynamics of drugs (Zhang and Finn, 2016).

A study has shown that an immune-related prognostic model provides new potential prognostic and therapeutic biomarkers (Wang et al., 2020), whereas few metabolismrelated genes were found. The prognostic value of the metabolism-based prognostic model that we constructed (3years AUC $=0.849$ ) was higher than that of the immune-based prognostic model (3-years $\mathrm{ROC}=0.711 ; 3$-years $\mathrm{ROC}=0.79$ ) (Wang et al., 2020; Zhang et al., 2020), indicating that the six metabolic mRNAs signature that we conducted had a robust high prognostic value. A recent study identified a four-gene metabolic signature which could efficiently stratify patients' OS (Liu et al., 2020). However, the prognostic model showed a low diagnostic performance in predicting long-term survival and little is known about the role and mechanisms of these metabolic genes (Liu et al., 2020). In this study we have constructed a gene signature composed of six metabolismrelated $m$ RNAs that has significant value in survival prediction and can be used to guide the adjuvant therapy for HCC.

\section{MATERIALS AND METHODS}

\section{Data Collection}

HCC transcriptome data and corresponding clinical information were downloaded from The Cancer Genome Atlas (TCGA) (https://portal.gdc.cancer.gov/) and International Cancer Genome Consortium (ICGC) (https://icgc.org). GSE22058, GSE25097, GSE45114, GSE62232, GSE121248, GSE76311 and GSE76427 were downloaded from the GEO database to test the expression of six metabolic mRNAs in tumor and normal control tissues. The KEGG data from the GSEA website (https://www.gsea-msigdb.org/) were downloaded for signaling pathway enrichment analysis.

\section{Samples Cohort}

Samples that lacked information on the survival time or survival status were eliminated. The TCGA dataset was randomly divided into a TCGA-training cohort $(n=186)$ and a TCGA-validation cohort $(n=187)$. A metabolic mRNA model of prognosis was established by using the TCGA-training cohort, TCGAvalidation cohort and ICGC cohort $(n=243)$ to test the model's predictive value of the model.

\section{Screening for Metabolic Gene Signature Related to Prognosis}

The R package "limma" was used to conduct differential analysis. The screening criteria were $|\log \mathrm{FC}|>0.5$ and a

\begin{tabular}{|c|c|c|c|c|c|}
\hline Clinical data & TCGA-training cohort & TCGA-validation cohort & Total & $x^{2}$ & $p$-value \\
\hline \multicolumn{6}{|l|}{ Gender } \\
\hline Female & 67 & 54 & 121 & 0.11 & 1.000 \\
\hline \multicolumn{6}{|l|}{ Age } \\
\hline$<60$ & 78 & 91 & 169 & & \\
\hline $\mathrm{G} 1+\mathrm{G} 2$ & 109 & 124 & 233 & & \\
\hline G3+G4 & 74 & 62 & 136 & 0.141 & 0.970 \\
\hline \multicolumn{6}{|l|}{ Survival status } \\
\hline Alive & 120 & 128 & 248 & & \\
\hline Dead & 67 & 59 & 126 & 0.222 & 1 \\
\hline \multicolumn{6}{|l|}{ TNM stage } \\
\hline $1+\|$ & 124 & 136 & 260 & & \\
\hline
\end{tabular}

TCGA: The Cancer Genome Atlas, Pearson $\chi^{2}$ test was used to explore random grouping results as appropriate. 

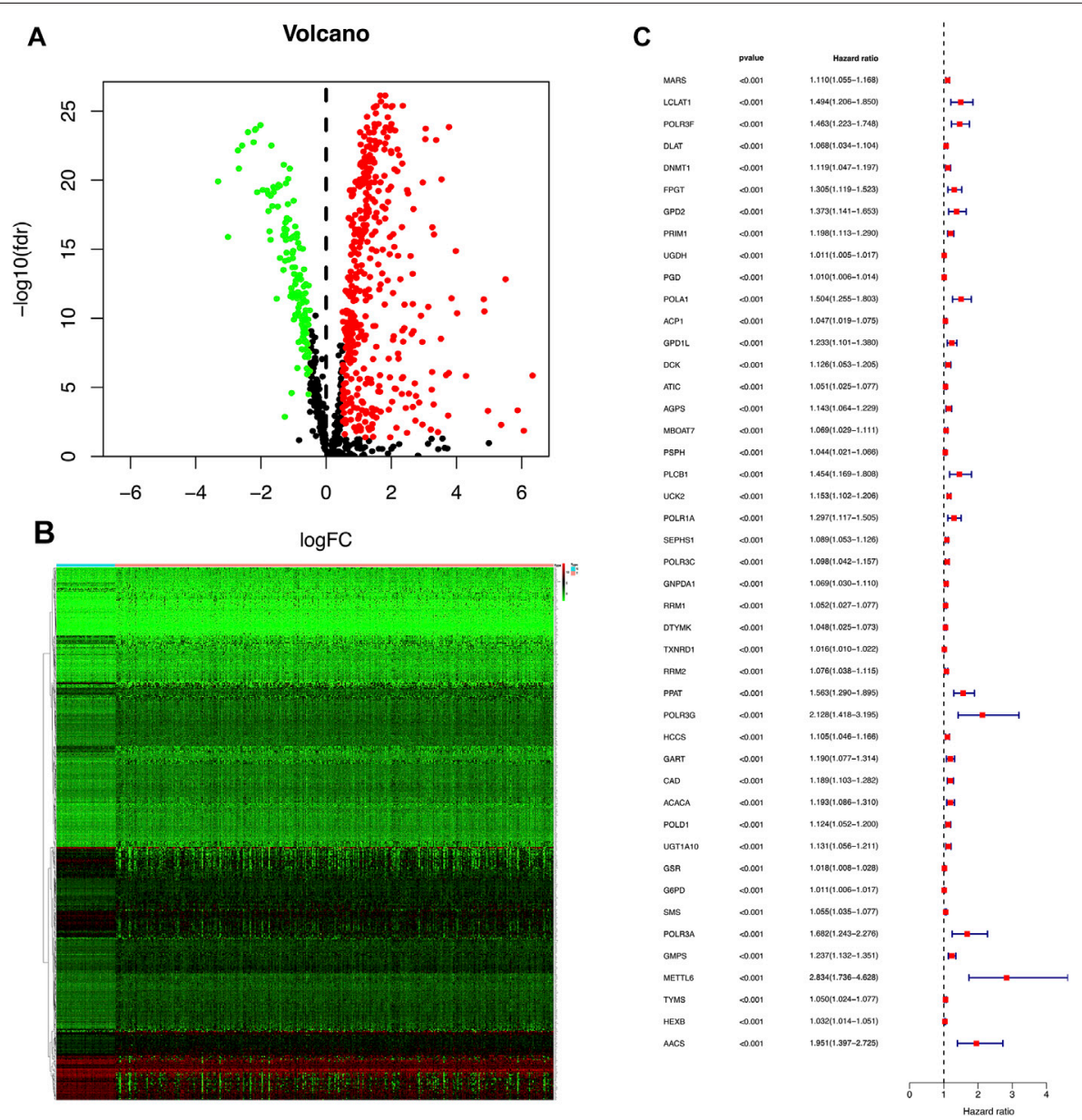

FIGURE 1 | (A) A volcano plot was used to show DEGs in TCGA HCC samples. Each point represents an mRNA. Red means a high expression level, green means low expression level, $|\log F C|>0.5$ and a $p$-value $<0.05$ are meaningful. FDR stands for false discovery rate. (B) A heatmap was used to show the expression of all differential genes in tumors and normal tissues. (C) Red represents high-risk DEGs, whereas green represents low-risk DEGs. The judgment of high and low risk was based on whether the HR value is greater than or equal to 1. There are 45 DEGs in the training cohort that are related to prognosis, and all are high-risk prognosis DEGs and have a $p$-value $<0.001$.

false discovery rate $($ FDR $)<0.05$. "Metabolism" was used as a key word to search all metabolic-related signaling pathways in KEGG. The gene signatures contained in these signaling pathways are considered to be metabolic-related gene signatures. We then intersected all differentially expressed genes (DEGs) with metabolic genes to arrive at all of the metabolic DEGs in HCC. We further screened the metabolic DEGs related to prognosis through Univariate Cox Regression analysis. For the process of analysis we used the R package "survival". The screening criteria were Hazard ratio $>1.000$ and $p$-value $<0.001$.

\section{Construction and Evaluation of a Metabolic mRNAs Prognosis Model}

The R package "glmnet" was used to build the least absolute shrinkage and selection operator (LASSO) Cox regression model of the metabolic DEGs related to prognosis. First, the risk value of each sample in the TCGA-training cohort was calculated using the following formula:

$$
\text { RiskScore }=\sum_{i}^{n}\left(\text { expression of } m R N A i * \operatorname{Coef}_{m R N A i}\right)
$$

Then we set the median as the threshold. By comparing the risk value with the threshold, the TCGA-training cohort was divided into a high-risk group and a low-risk group. Second, the same method was used to divide the TCGA-validation cohort and the ICGC-validation cohort into two groups, with a high and low risk respectively. The threshold of each validation cohort was the same as the threshold of the TCGA-training cohort. Third, the $\mathrm{R}$ package "survival" was used to test whether there were prognostic differences between the high and low-risk groups in the three cohorts. Fourth, the relationship between clinical features and the risk score was calculated through univariate independent prognostic analysis (UIPA) and multivariate independent prognostic analysis 


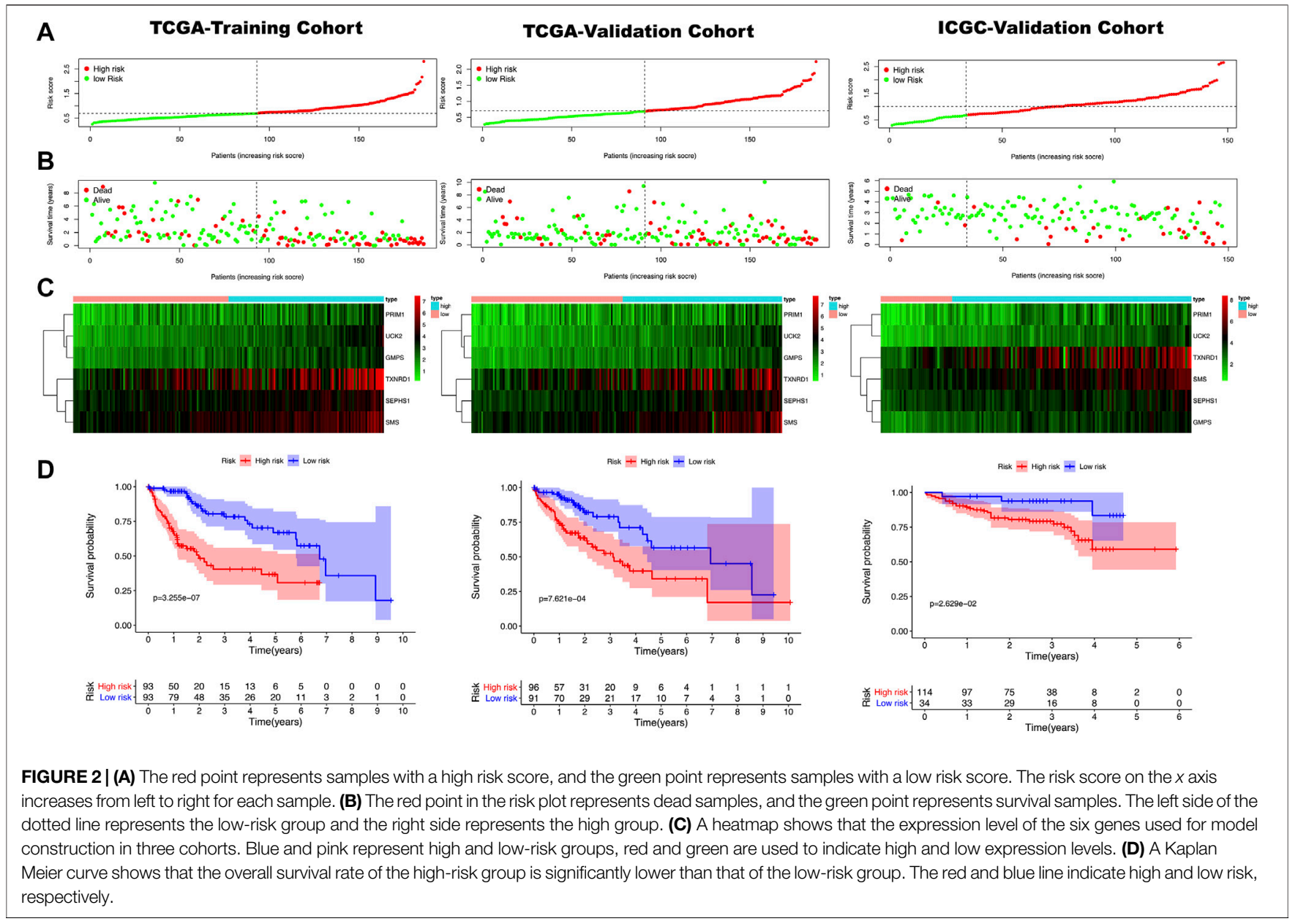

(MIPA). Fifth, the area under the curve (AUC) and the timedependent Receiver Operating Characteristic (ROC) curves were assessed to evaluate the predictive power of the risk score for survival prediction.

\section{Building and Validating a Predictive Nomogram}

We used the R package "rms" to draw a nomogram that could be used to predict the prognosis of HCC patients. Each clinical indicator corresponded to a score. The patient's clinical characteristics in the nomogram were filled in to get the corresponding score, and the score of each indicator was added to obtain the total score. Variables with no clinical significance were not included in the nomogram. The total score was compared with the 1-, 3-, and 5-years survival probability scale to estimate the patient's possible survival time.

\section{Validation of Prognostic mRNA Signature Expression}

In order to further verify the expression of mRNA signatures in HCC, 7 HCC datasets were downloaded from the Gene
Expression Omnibus (GEO) database (https://www.ncbi.nlm. nih.gov/geo/), including GSE22058 (tumor: normal = 100:97), GSE25097 (tumor: normal = 268:243), GSE45114 (tumor: normal = 24:25), GSE62232 (tumor: normal = 81:10), GSE76311 (tumor: normal = 62:59), GSE76427 (tumor: normal $=115: 52$ ) and GSE121248 (tumor: normal $=70: 37$ ). We retrieved the immunohistochemical staining (IHS) results of the proteins corresponding to these mRNA signatures from the human protein atlas (https://www.proteinatlas.org/, HPA) to obtain the protein expressions. The overall survival was analyzed using GEPIA (http://gepia.cancer-pku.cn/) (Tang et al., 2017).

\section{Multiple Enrichment Analysis of Risk mRNA Signatures}

The original mRNA matrix of three cohorts was divided into a highrisk group and a low-risk group. The two groups were subjected to gene enrichment analysis to obtain the top 5 significantly enriched metabolic signaling pathways. Enrichment analysis was performed using gene-set enrichment analysis software (GSEA_4.0.3). The dataset selected for analysis was KEGG. Each enrichment was carried out for 1,000 cycles, and the R packages "plyr", "ggplot2", "grid" and "gridExtra" were used to draw multiple GSEA plots. 


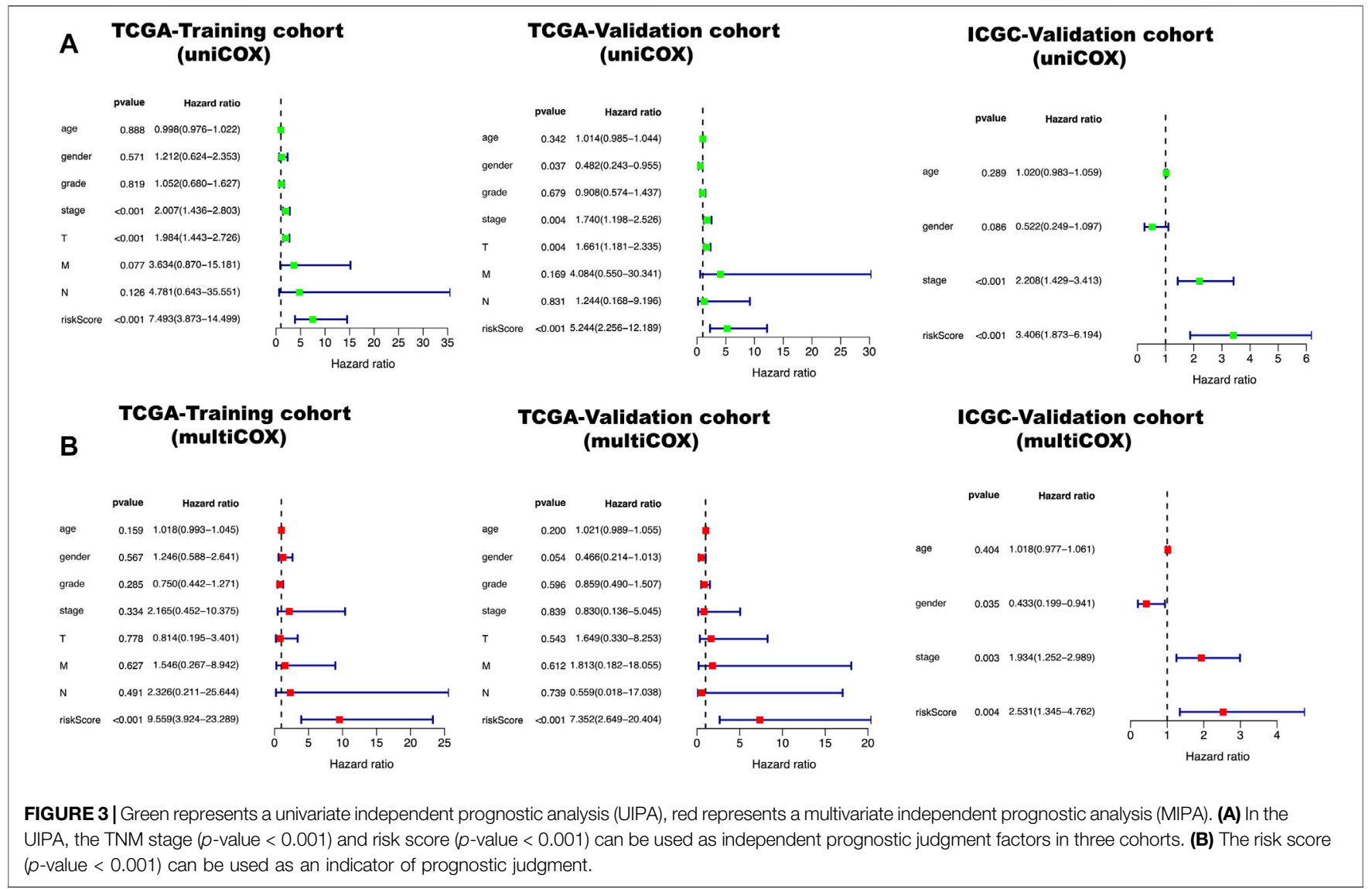

\section{Statistical Analysis}

Statistical analyses were performed using SPSS (version 22.0) and R (version 3.6.1 for Mac). The Wilcox test was used in the difference analysis process. If there were multiple lines of a gene in the expression dataset, they were averaged into one line. Pearson $\chi^{2}$ test was used to explore random grouping results as appropriate. Except for the correlation coefficient of the model, all numerical values retained three digits after the decimal point. Unless otherwise specified, a $p$-value $<0.05$ is considered statistically significant.

\section{RESULTS}

\section{Screening for 45 DEGs Associated With Poor Prognosis}

Clinical information of the TCGA training cohort and TCGA validation cohort is shown in Table 1. There was no difference in clinical characteristics between the TCGA-training cohort and TCGA-validation cohort in a chi-square test (Table 1). By comparing the HCC tissues in TCGA with the adjacent tissues, we obtained 547 DEGs. Among them, 137 were downregulated and 410 were up-regulated. Differential expression results were visualized in Figure 1A. The log fold change (log FC) and expression level of all DEGs were in Supplementary Table S1. Subsequently, the 547 DEGs were further screened in the training cohort through survival analysis and 45 DEGs related to prognosis were obtained. These 45 genes were all high-risk mRNAs, and their expression levels were negatively correlated with the prognosis of patients. A heatmap was used to show the expression of all differential genes in tumors and normal tissues in Figure 1B. All of the prognostic DEGs were shown in Figure 1C. Supplementary Table S2 included the Hazard ratio (HR), HR.95L and HR.95H value of each mRNAs.

\section{Construction of a Prognostic Models Composed of Six mRNA Signatures}

We put the 45 mRNA signatures obtained in the previous step into the training cohort for model construction. Only six mRNAs were ultimately used to construct the model, i.e. primase polypeptide (PRIM1), uridine-cytidine kinase 2 (UCK2), selenophosphate synthetase 1 (SEPHS1), thioredoxin reductase (TXNRD1), spermine synthase (SMS) and granulocyte-monocyte progenitors (GMPS). The correlation coefficient of each mRNA was shown in Supplementary Table S3. The result was the following model: (expression of PRIM1 * 0.0036) + (expression of UCK2 * 0.0498) + (expression of SEPHS11 * 0.0087) + (expression of TXNRD1 * 0.0029)+ (expression of SMS * $0.0109)+\left(\right.$ expression of $\left.\mathrm{GMPS}^{\star} 0.0207\right)$. This formula was used to calculate the risk score of each sample in the training cohort with the medium score 0.692 as the threshold. Then, 93 


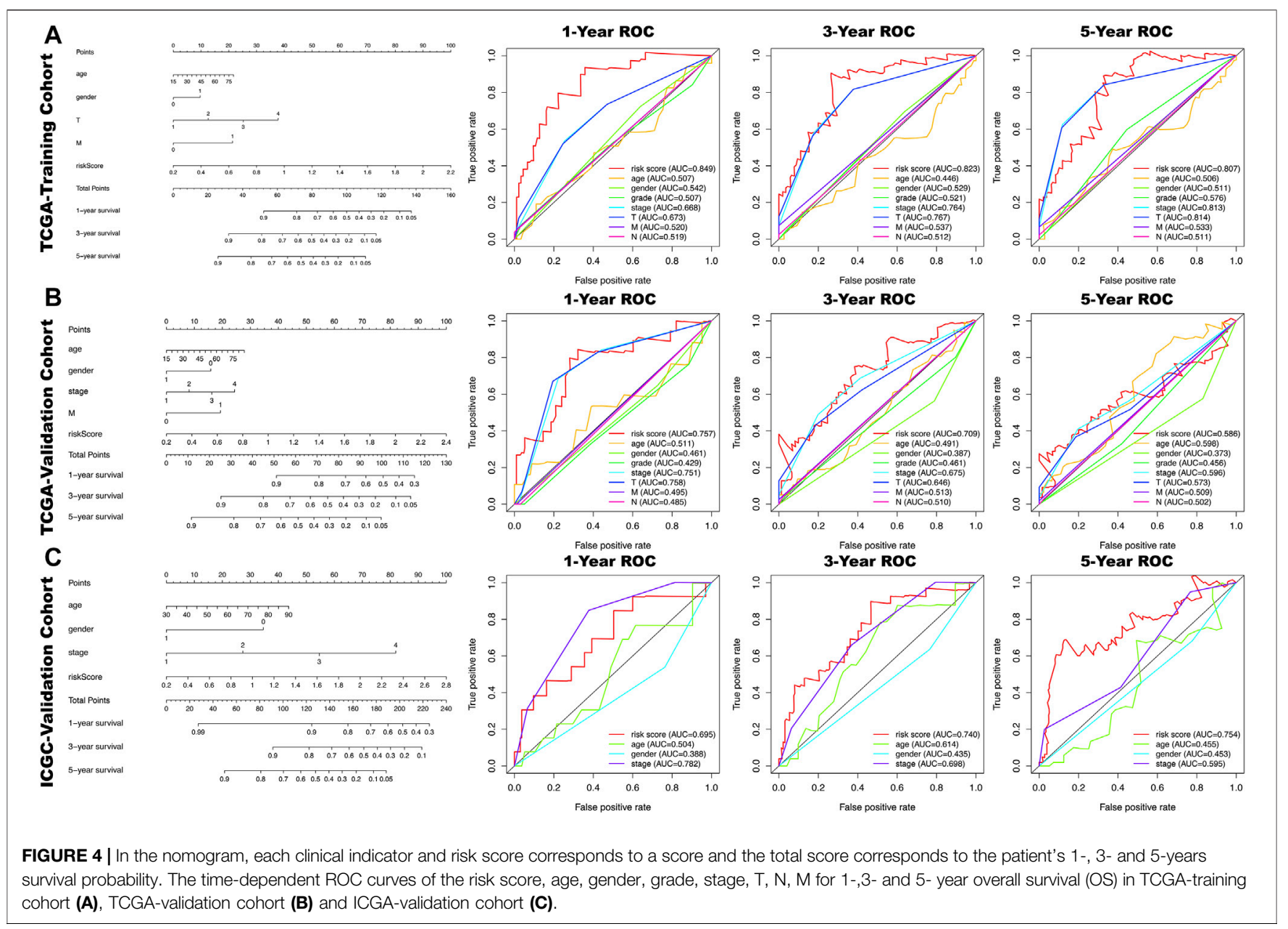

cases were allocated to high-risk group and 93 cases to the lowrisk group. Using the same formula and threshold, we ended up with 96 samples in the high-risk group and 91 samples in the low-risk group in the TCGA-validation cohort, and 114 samples in the high-risk group and 34 samples in the low-risk group in the ICGC-validation cohort. The overall grouping results tended to be average (Figure 2A Supplementary Table S4). The risk curve shown in Figure 2B indicated that as the risk score increased, the number of deaths also increased. The results of the differential analysis between the high and low risk groups showed that the expression levels of these six genes in the high-risk group were higher than in the low-risk group (Figure 2C). Kaplan Meier curve showed that the prognosis of the high-risk group was significantly worse than that of the low-risk group (Figure 2D).

\section{The Model Could Predict HCC Patient Survival}

All clinical characteristics and risk scores were subjected to a univariate independent prognostic analysis (UIPA). The UIPA results showed that TNM stage $(p$-value $<0.001)$ and risk score $(p$-value $<0.001)$ could be used as independent prognostic judgment factors, and the results were validated in the training cohort and the two validation cohorts, respectively (Figure 3A).
MIPA analysis showed that only the risk score could be used as an independent prognostic judgment factor (Figure 3B). The Hazard ratio (HR), HR.95L and HR.95H value of each mRNA were listed in Supplementary Table S5. The nomogram used to evaluate the 1-, 3- and 5-years survival rate of patients was shown in Figure 4A. In the results of the ROC curve of the training cohort (Figure 4B), the Area Under Curve (AUC) of the risk score was 0.849 and the value for judging the prognosis far exceeded that of the indicators such as age, gender, pathological grade and TNM stage. In the two validation cohorts, the risk score also performed well (TCGA-AUC = 0.757, ICGC-AUC = 0.695).

\section{Verification of Expression Levels of Six mRNAs}

The six mRNAs i.e. PRIM1, UCK2, SEPHS1, TXNRD1, SMS and GMPS, all had higher expression levels in the HCC sample than in normal controls, which was consistent in the eight datasets. What's more, the samples with high expression levels had lower overall survival rates than the samples with low expression levels in the TCGA dataset (Figure 5A). A violin chart shows the relationship between gene expression level and 

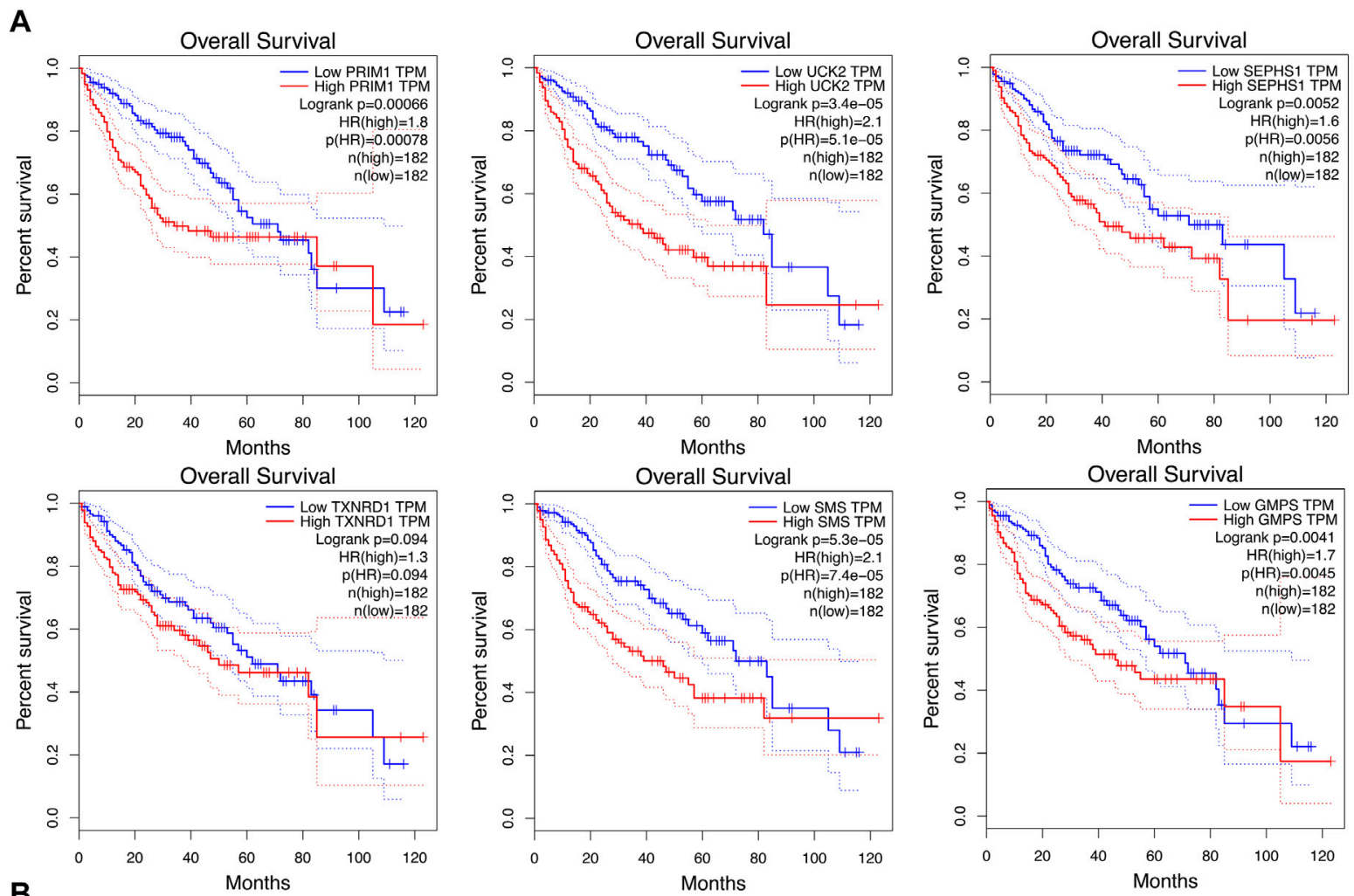

B
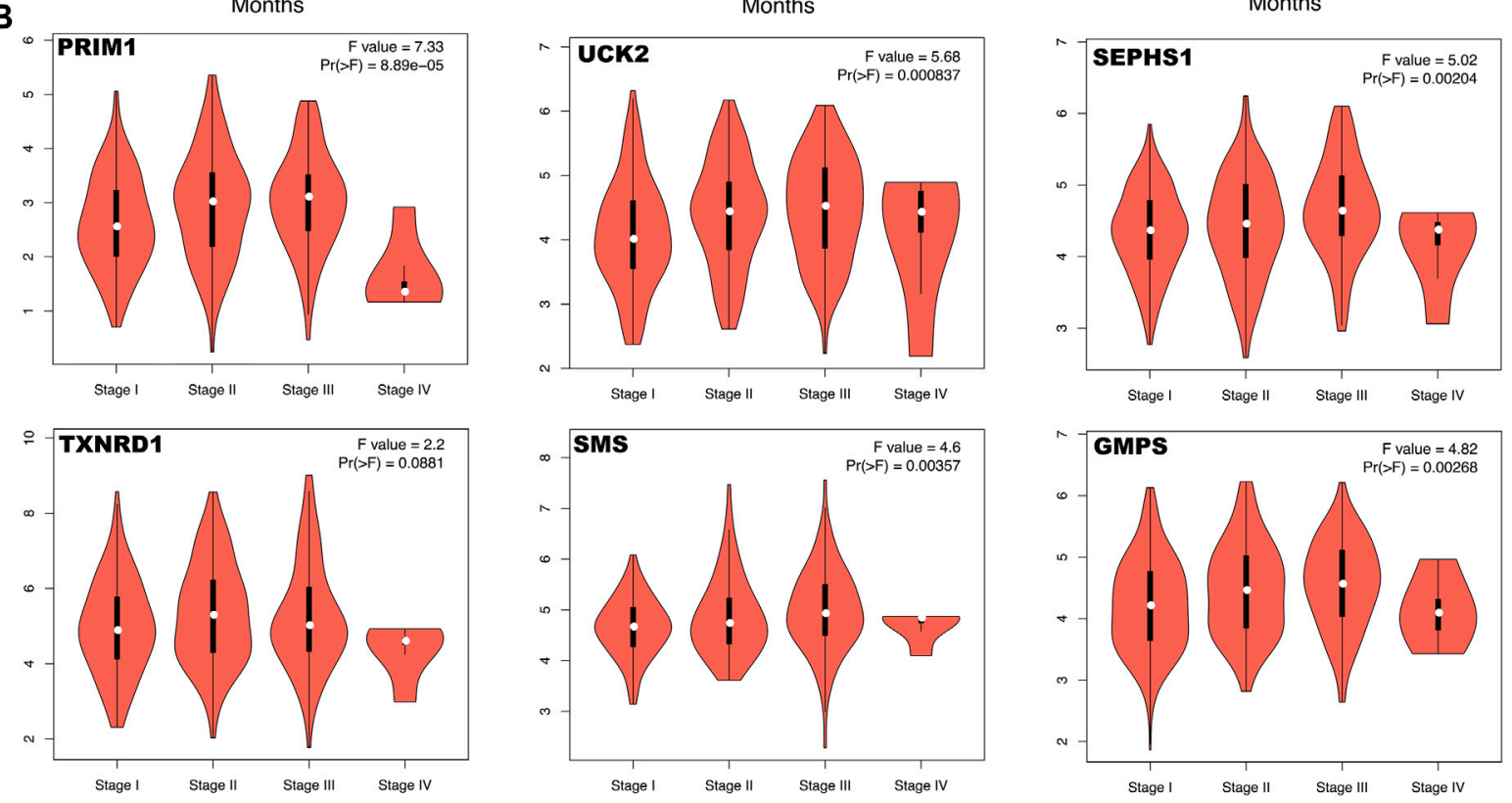

FIGURE 5 | (A) Kaplan Meier curve shows that the overall survival rate of the high expression levels of the six mRNA signatures are significantly lower than that of the low expression levels. (B) Violin chart shows the relationship between gene expression level and clinical stage.

clinical stage (Figure 5B). The HPA database contained only the immunohistochemical results of PRIM1, SEPHS1, TXNRD1 and SMS. The results showed that the proteins corresponding to four mRNA signatures had high expression level in HCC cells compared with normal hepatocytes (Figure 6A). Except for the $p$-value of SMS in GSM62232, which was larger than 0.05 , all other results have a $p$-value $<0.05$, which was statistically significant (Figure 6B).

\section{GSEA Enrichment Analysis Results}

The enrichment analysis results included many metabolic-related signaling pathways. We selected the 5 most significantly related 


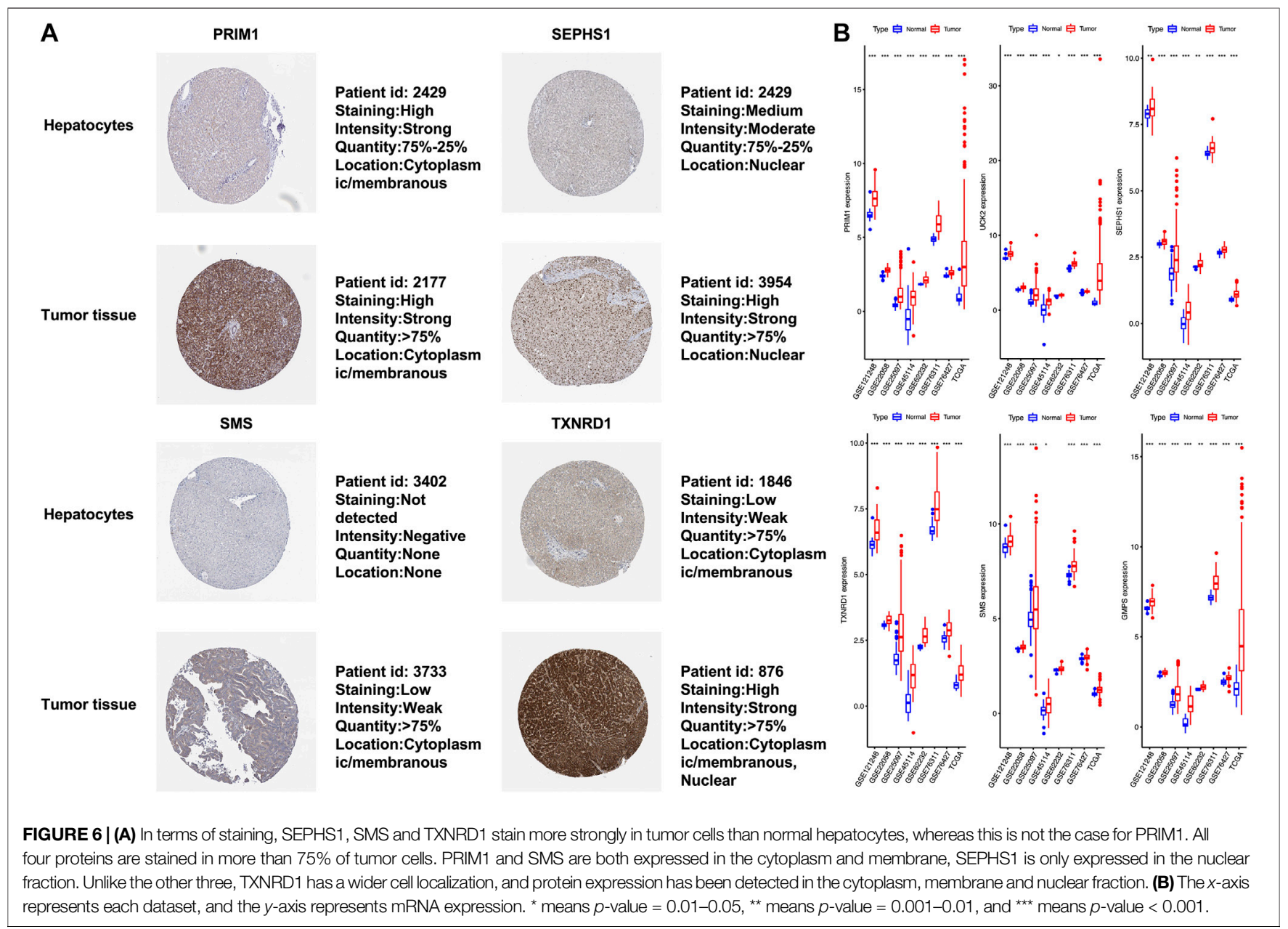

signaling pathways in the high and low-risk groups for display in Figure 7, whereas the relevant enrichment scores are shown in Table 2-4. The KEGG_PYRIMIDINE_METABOLISM and KEGG_PURINE_METABOLISM were two abundant metabolic pathways in high-risk scoring. The most significant enrichment in the low-risk grouping was the KEGG_FATTY_ACID_METABOLISM signal pathway.

\section{Evaluation of Prognostic Model Efficacy Compared to ABIC, ALBI Score, ALBI Grade}

The time-dependent ROC curves of risk score, age, gender, grade, stage, T, N, M, ABIC, ALBI, ALBI grade, AFP for 1 year DFS and OS in the TCGA-training cohort and TCGA-validation cohort were determined. The results show that the predictive value of OS and DFS in the training cohort of the prognostic model based on the six metabolic genes is higher than for the above-mentioned metabolic predictors (Figure 8).

\section{DISCUSSION}

We constructed a gene signature composed of six metabolismrelated mRNAs to investigate its role in HCC. We found six metabolism-related mRNAs in HCC through the LASSO regression model by analyzing the data from public datasets. This model performed well in predicting the prognosis of HCC patients, especially for OS within 3 and 5 years. Two articles were published that focused on the immune-based prognosis model of liver cancer (Wang et al., 2020; Zhang et al., 2020). The prognostic value of the metabolism-based prognostic model that we constructed (3-years AUC $=0.849$ ) was higher than that of the immune-based prognostic model (3-years ROC $=0.711$; 3 years $\mathrm{ROC}=0.79)$, indicating that the six metabolic mRNAs signature that we constructed had a robust high prognostic value.

A recent study identified a four-gene metabolic signature and the signature that could efficiently stratify patients' OS(Liu et al., 2020). The efficacy of our model was better than of the previous model, based on the following arguments. First, the prognostic value of our prognostic model in TCGA-training cohort (3-years AUC $=0.849$ ) was higher than Liu's ( 3 -years ROC $=0.71$ ), as was true for the TCGA-validation cohort $(\mathrm{AUC}=0.757$ vs $\mathrm{AUC}=$ 0.70 ). Second, we first tested the efficacy of our model internally in the TCGA data and then externally verified it in the Japanese cohort in the ICGC database. As an internationally cooperative tumor database, the ICGC database includes HCC cancer data that is more reliable and comprehensive. The Japanese cohort we 


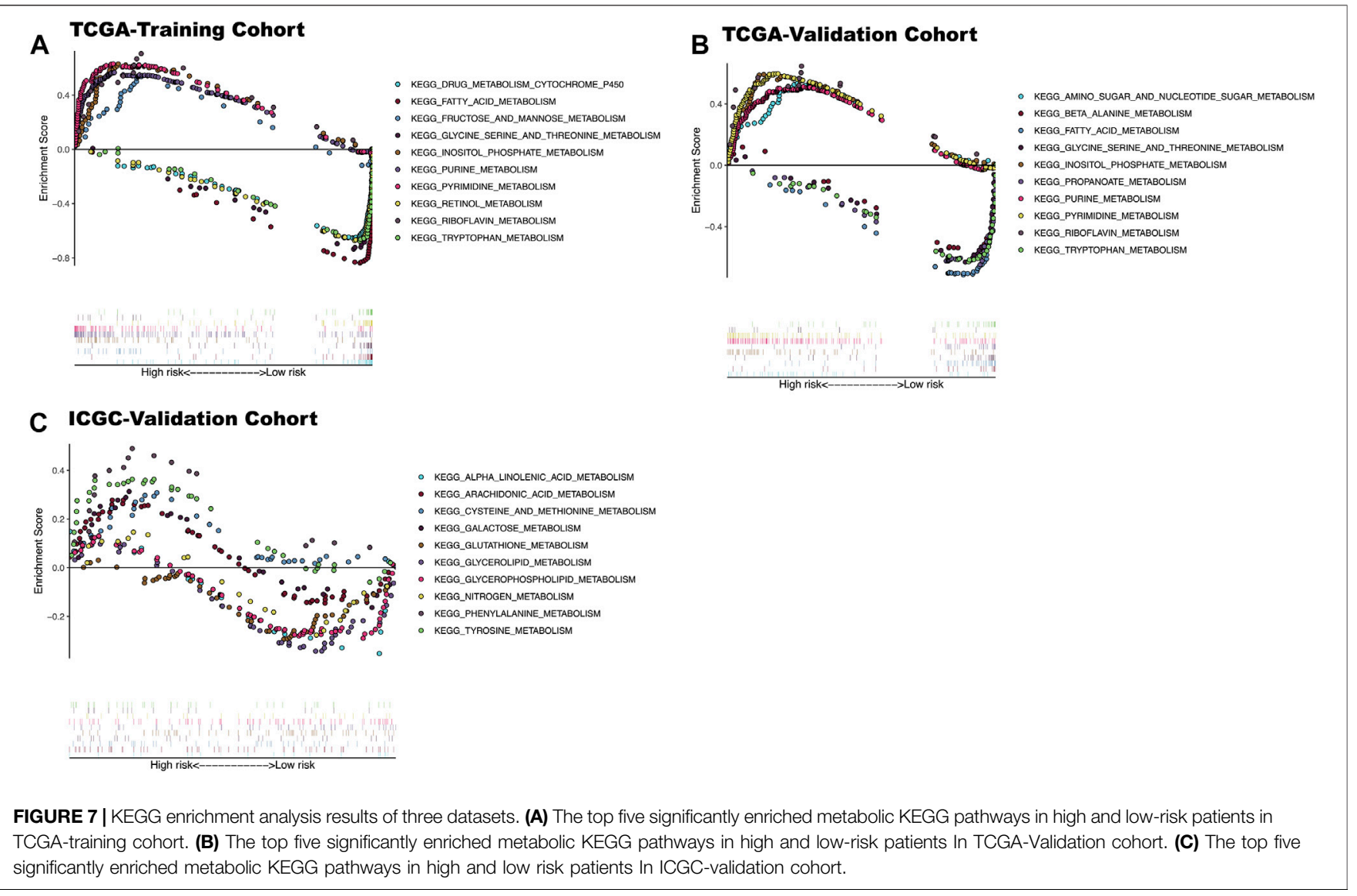

TABLE 2 | Top 5 metabolic signaling pathways significantly enriched in high/low risk groups of the training cohort.

\begin{tabular}{|c|c|c|c|c|c|c|}
\hline No & Signaling pathways & Risk & ES & NES & $\begin{array}{l}\text { Nom } \\
p \text {-val }\end{array}$ & $\begin{array}{l}\text { FDR } \\
q \text {-va }\end{array}$ \\
\hline 1 & KEGG_PYRIMIDINE_METABOLISM & High & 0.63 & 1.99 & 0.000 & 0.004 \\
\hline 2 & KEGG_PURINE_METABOLISM & High & 0.57 & 1.97 & 0.000 & 0.003 \\
\hline 4 & KEGG_INOSITOL_PHOSPHATE_METABOLISM & High & 0.63 & 1.77 & 0.004 & 0.019 \\
\hline 5 & KEGG_FRUCTOSE_AND_MANNOSE_METABOLISM & High & 0.54 & 1.74 & 0.010 & 0.022 \\
\hline 6 & KEGG_FATTY_ACID_METABOLISM & Low & -0.85 & -2.03 & 0.000 & 0.002 \\
\hline 9 & KEGG_TRYPTOPHAN_METABOLISM & Low & -0.69 & -1.92 & 0.002 & 0.004 \\
\hline 10 & KEGG_DRUG_METABOLISM_CYTOCHROME_P450 & Low & -0.66 & -1.90 & 0.000 & 0.005 \\
\hline
\end{tabular}

Abbreviations: ES, enrichment score; NES, normalized enrichment score; Nom p-val, nominal p-value; FDR q-val, false discovery rate q-value.

used for external verification included 243 liver cancer cases, which was higher than Liu's 215 cases in GSE14520. What's more, it has test power in the Japanese cohort, which shows that our model is also applicable to Asian races, and the external verification data used by Liu is the same as the TCGA data from the United States. Third, we did a differential expression analysis of the six genes used to construct the model in seven external HCC data (a total of 1,243 cases, tumor vs. normal $=720$ vs. 523), and the results showed that the six genes were all highly expressed in liver cancer. Liu's research did not test the expression level of genes used to construct the model in other databases.
Fourth, Liu's model did not provide a relationship between the proposed model and DFS.

AFP is currently the most commonly used metabolic biomarker in clinical practice (Notarpaolo et al., 2017; Pinyol et al., 2019). It is often used to predict the prognosis of patients. We added AFP to compare it with our prediction model. The results suggested that our predictive model had a higher predictive value than AFP for both OS and DFS, whether it is in the training or validation cohort. Furthermore, we used same method to evaluate the efficacy of the prognostic model compared to Age-Bilirubin-International Normalized Ratio-Creatinine (ABIC) (Mitchell et al., 2017), the 
TABLE 3 | Top 5 metabolic signaling pathways significantly enriched in high/low risk groups of the TCGA-Validation cohort.

\begin{tabular}{|c|c|c|c|c|c|c|}
\hline No & Signaling pathways & Risk & ES & NES & $\begin{array}{l}\text { Nom } \\
p \text {-val }\end{array}$ & $\begin{array}{l}\text { FDR } \\
q \text {-val }\end{array}$ \\
\hline 1 & KEGG_PYRIMIDINE_METABOLISM & High & 0.60 & 1.69 & 0.000 & 0.308 \\
\hline 2 & KEGG_PURINE_METABOLISM & High & 0.51 & 1.53 & 0.007 & 0.226 \\
\hline 3 & KEGG_RIBOFLAVIN_METABOLISM & High & 0.65 & 1.47 & 0.038 & 0.209 \\
\hline 4 & KEGG_INOSITOL_PHOSPHATE_METABOLISM & High & 0.59 & 1.45 & 0.026 & 0.202 \\
\hline 5 & KEGG_AMINO_SUGAR_AND_NUCLEOTIDE_SUGAR_METABOLISM & High & 0.53 & 1.41 & 0.044 & 0.230 \\
\hline 6 & KEGG_TRYPTOPHAN_METABOLISM & Low & -0.64 & -1.65 & 0.004 & 0.080 \\
\hline 7 & KEGG_FATTY_ACID_METABOLISM & Low & -0.73 & -1.64 & 0.020 & 0.064 \\
\hline 8 & KEGG_PROPANOATE_METABOLISM & Low & -0.68 & -1.55 & 0.051 & 0.119 \\
\hline 9 & KEGG_BETA_ALANINE_METABOLISM & Low & -0.63 & -1.53 & 0.048 & 0.124 \\
\hline 10 & KEGG_GLYCINE_SERINE_AND_THREONINE_METABOLISM & Low & -0.65 & -1.48 & 0.060 & 0.145 \\
\hline
\end{tabular}

Abbreviations: ES, enrichment score; NES, normalized enrichment score; Nom p-val, nominal p-value; FDR q-val, false discovery rate q-value.

\begin{tabular}{|c|c|c|c|c|c|c|}
\hline No & Signaling pathways & Risk & ES & NES & $\begin{array}{l}\text { Nom } \\
p \text {-val }\end{array}$ & $\begin{array}{l}\text { FDR } \\
q \text {-val }\end{array}$ \\
\hline 1 & KEGG_PHENYLALANINE_METABOLISM & High & 0.49 & 1.23 & 0.246 & 1 \\
\hline 2 & KEGG_CYSTEINE_AND_METHIONINE_METABOLISM & High & 0.31 & 1.06 & 0.371 & 1 \\
\hline 3 & KEGG_TYROSINE_METABOLISM & High & 0.36 & 1.02 & 0.440 & 1 \\
\hline 4 & KEGG_GALACTOSE_METABOLISM & High & 0.31 & 1 & 0.461 & 1 \\
\hline 5 & KEGG_ARACHIDONIC_ACID_METABOLISM & High & 0.28 & 0.95 & 0.541 & 1 \\
\hline 6 & KEGG_ALPHA_LINOLENIC_ACID_METABOLISM & Low & -0.44 & -1.34 & 0.119 & 1 \\
\hline 7 & KEGG_GLYCEROLIPID_METABOLISM & Low & -0.36 & -1.28 & 0.164 & 1 \\
\hline 8 & KEGG_GLYCEROPHOSPHOLIPID_METABOLISM & Low & -0.31 & -1.16 & 0.276 & 1 \\
\hline 9 & KEGG_GLUTATHIONE_METABOLISM & Low & -0.30 & -1.05 & 0.369 & 1 \\
\hline 10 & KEGG_NITROGEN_METABOLISM & Low & -0.31 & -1.00 & 0.458 & 1 \\
\hline
\end{tabular}

Abbreviations: ES, enrichment score; NES, normalized enrichment score; Nom p-val, nominal p-value; FDR q-val, false discovery rate q-value.

albumin-bilirubin (ALBI) score and ALBI grade (Mitchell et al., 2017). The results showed that the predictive value of OS and DFS in the training cohort of the prognostic model based on the six metabolic genes is higher than that of the above-mentioned metabolic predictors. Our nomogram has increased the testing power of clinical information, making its predictive power more accurate.

The advantage of the LASSO regression model is that it offers the possibility to delete genes that are highly correlated in order to reduce errors. Therefore, highly relevant mRNAs such as METTL6, POLR3A, POLR3G, AACS, etc. were not included when we constructed our model. The pathological grade and TNM stage are commonly used to predict the prognosis of HCC patients. In addition to the grade indicator, risk score, and TNM staging, these results were consistent with the training queue. The deviation of grade results had little effect on the model. In survival analysis, only TXNRD1 was not statistically significant, although the $p$-value was close to 0.05 . When we reviewed the original data, we found that two of the samples with high expression of TXNRD1 had extremely long survival times, and the remaining patients had generally low survival times. The Kaplan-Meier (KM) graph also showed that the overall survival rate of patients with a high expression of TXNRD1 was lower than those with a low expression. This could be due to a sampling error, but when TXNRD1 and five other genes were used to form a model, it was statistically significant in predicting the survival rate, and its $p$-value was less than 0.001 . In terms of gene expression verification, the size of the sample included in the differential analysis was large enough with high credibility. The prognostic model in our study has a high predictive accuracy with an AUC as high as 0.849 . In addition, it showed a good performance in the verification set of two different databases, reflecting the stability of the model.

In the results of the enrichment analysis, we showed the top 5 significantly enriched metabolism-related signaling pathways. The KEGG_PYRIMIDINE_METABOLISM was the most abundant metabolic pathway in high risk scoring. Pyrimidine metabolism was very important in the process of cell proliferation. In HCC, the activation of the PYRIMIDINE signaling pathway could promote liver enlargement and tumor cell proliferation (Cox et al., 2016). The most significant enrichment in the low-risk grouping was the KEGG_FATTY_ACID_METABOLISM signal pathway. Lipid metabolism is one of the main sources of energy for tumor cells, which is very helpful for the adaptation of tumor cells to the microenvironment (Sangineto et al., 2020). The rest of the signaling pathways such as KEGG GLYCINE SERINE AND THREONINE METABOLISM (Li et al., 2015), KEGG_ FRUCTOSE_AND_MANNOSE_METABOLISM (Huang et al., 2019b) and KEGG_DRUG_METABOLISM_CYTOCHROME_ P450 (Nekvindova et al., 2020) have been reported in literature to be related to the malignant phenotype of HCC. These studies 

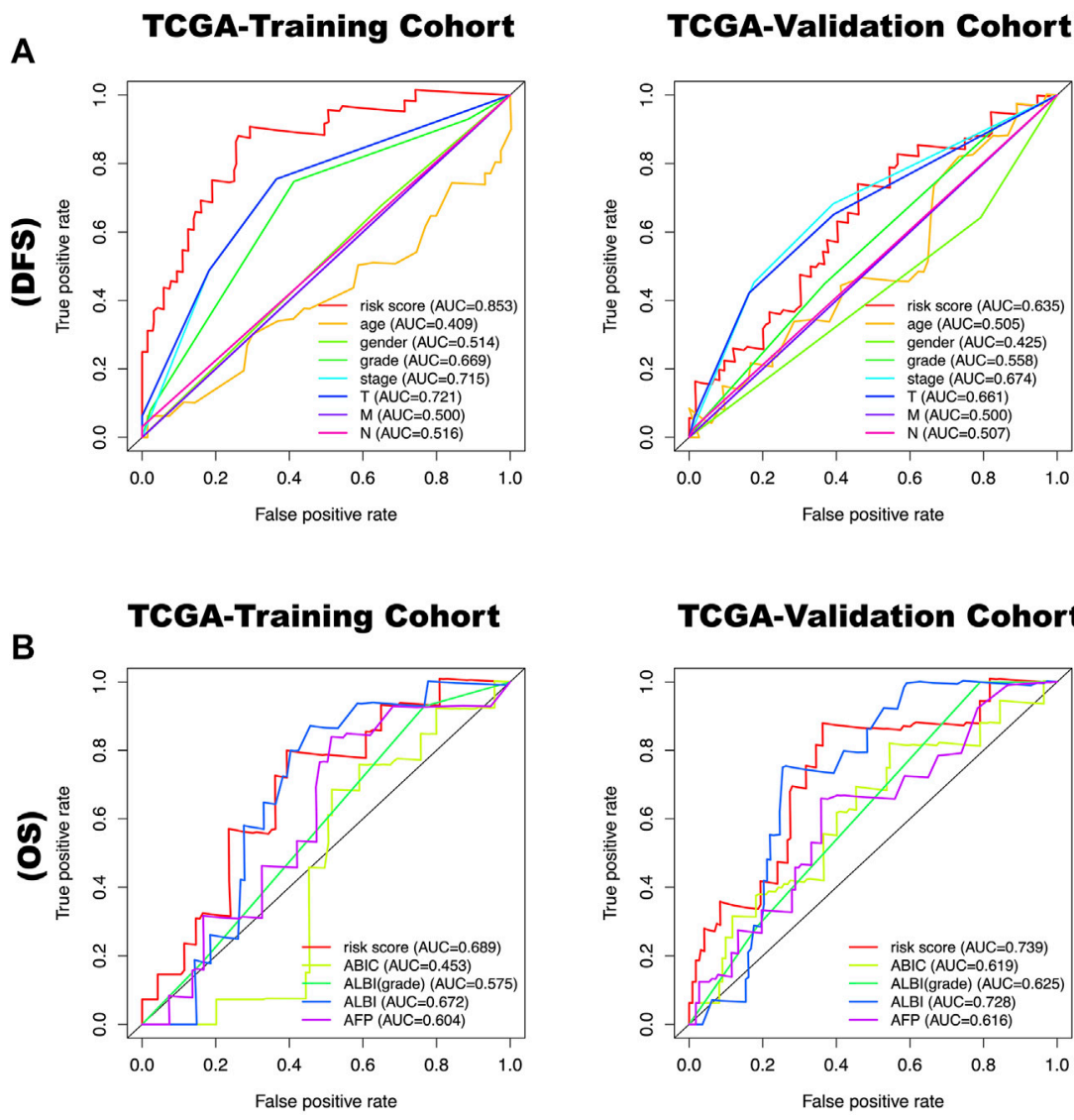

\section{TCGA-Validation Cohort}

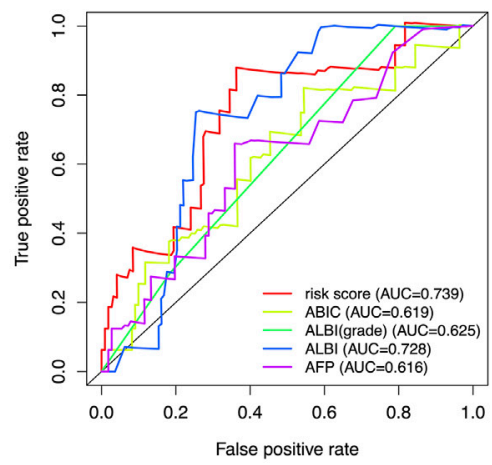

TCGA-Training Cohort

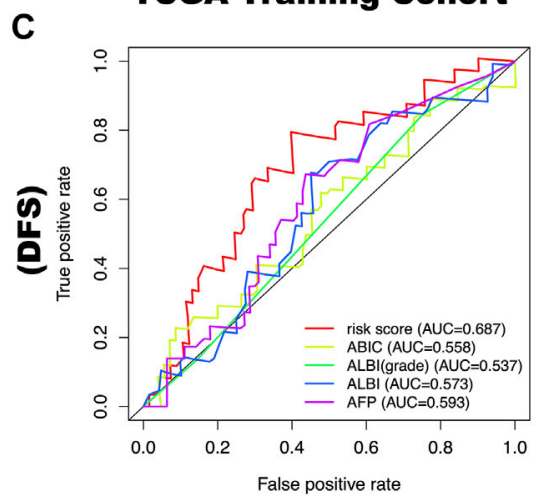

TCGA-Validation Cohort

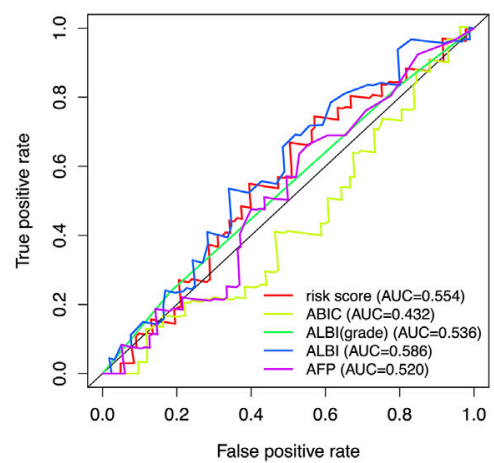

FIGURE 8 | (A) The time-dependent ROC curves of risk score, age, gender, grade, stage, T, N, M for 1 year disease-free survival (DFS) in the TCGA-training cohort and TCGA-validation cohort. (B) The time-dependent ROC curves of risk score, ABIC, ALBI, ALBI grade, AFP for 1-year overall survival (OS) in the TCGA-training cohort and TCGA-validation cohort. (C) The time-dependent ROC curves of risk score, ABIC, ALBI, ALBI grade, AFP for 1-year disease-free survival (DFS) in the TCGA-training cohort and TCGA-validation cohort.

reflect that the six mRNAs in our model are closely related to HCC metabolism.

PRIM1 hasn't been reported in liver cancer (Xu et al., 2016). However, in breast cancer and lung cancer it was found that the expression level of PRIM1 in tumor tissue was higher (Lee et al., $2019 b$ ). We think it is necessary to carry out more in-depth mechanism research to explore its mode of action in liver cancer. Like PRIM1,
GMPS was found to be expressed higher in tumor tissues and peripheral blood of liver cancer patients (Zhang et al., 2017; Yin et al., 2019). UCK2 has been reported to be highly expressed in HCC tumors It promotes tumor cell metastasis and invasion through the Stat3 pathway (Zhou et al., 2018) and can be used as an independent prognostic factor (Huang et al, 2019a). TXNRD1 works as an oncogene in HCC and an TXNRD1 inhibitor significantly inhibits 
tumor growth (Fu et al., 2017; Lee et al., 2019a). TXNRD1 is an antioxidant enzyme regulated by the Nrf2/Keap1 pathway and it's expression level is also negatively regulated by the non-coding RNA miR_125b_5p (Tuo et al., 2018; Hua et al., 2019). Tuo et al. found that TXNRD1 inhibitors can enhance the sensitivity of HCC tumor cells to sorafenib. In the future, an inhibitor suitable for clinical use could be developed for TXNRD1 and its combined use with sorafenib might improve the therapeutic effect. SMS, a polyamine biosynthetic enzyme, is overexpressed in patients with colorectal cancer and collaborates with MYC to promote colorectal cancer cell survival (Guo et al., 2020). However, little is known about SMS in liver disease and HCC and more experiments are necessary to reveal its role in HCC. Selenophosphate synthetase 2 (SEPHS 2) is essential for survival of cancer (Carlisle et al., 2020), but little research has focused on the role of SEPHS 1 in cancer and more functional experiments are needed.

Our study has some limitations. First, all of the data in our research come from databases, and the mRNA expression level and corresponding protein expression of the six genes for construction of the model need further clinical specimen verification. In addition, although our model can predict the prognosis of patients, there are many factors that affect the prognosis of HCC patients, such as tumor immunity, non-coding RNA, etc. Although our model has been well validated in the two large databases-TCGA and ICGC-we still need more data to fully verify the model's reliability. Further experiments are required to verify the relationship between the six genes used to construct the model and the metabolism-related signaling pathways we obtained through enrichment analysis and their mechanism in the progression of HCC. What's more, due to missing data for tumor number, BCLC staging and treatment type in TCGA, we can't evaluate the efficacy of the prognostic model compared to the above-mentioned three variables.

In conclusion, we established a gene signature composed of six metabolism-related mRNAs that has significant value in predicting HCC survival and can be used to guide the adjuvant therapy for HCC.

\section{REFERENCES}

Best, J., Bechmann, L. P., Sowa, J. P., Sydor, S., Dechene, A., Pflanz, K., et al. (2020). GALAD score detects early hepatocellular carcinoma in an international cohort of patients with nonalcoholic steatohepatitis. Clin. Gastroenterol. Hepatol. 18 (3), 728-735. doi:10.1016/j.cgh.2019.11.012

Beyoglu, D., and Idle, J. R. (2020). Metabolomic and lipidomic biomarkers for premalignant liver disease diagnosis and therapy. Metabolites 10 (2), 50. doi:10. 3390/metabo10020050

Bray, F., Ferlay, J., Soerjomataram, I., Siegel, R. L., Torre, L. A., and Jemal, A. (2018). Global cancer statistics 2018: GLOBOCAN estimates of incidence and mortality worldwide for 36 cancers in 185 countries. CA: A Cancer J. Clin. 68 (6), 394-424. doi:10.3322/caac.21492

Carlisle, A. E., Lee, N., Matthew-Onabanjo, A. N., Spears, M. E., Park, S. J., Youkana, D., et al. (2020). Selenium detoxification is required for cancer-cell survival. Nat. Metab. 2 (7), 603-611. doi:10.1038/s42255-020-0224-7

Cox, A. G., Hwang, K. L., Brown, K. K., Evason, K., Beltz, S., Tsomides, A., et al. (2016). Yap reprograms glutamine metabolism to increase nucleotide biosynthesis and enable liver growth. Nat. Cell Biol. 18 (8), 886-896. doi:10. 1038/ncb3389

Dai, T. X., Deng, M. B., Ye, L. S., Liu, R. Q., Lin, G. Z., Chen, X. L., et al. (2020). Prognostic value of combined preoperative gamma-glutamyl transpeptidase to

\section{DATA AVAILABILITY STATEMENT}

The original contributions presented in the study are included in the article/Supplementary Material, further inquiries can be directed to the corresponding author/s. Global list :Gene Expression Omnibus, https://portal.gdc.cancer.gov/, https://www. ncbi.nlm.nih.gov/, https://www.ncbi.nlm.nih.gov/geo/, https:// www.proteinatlas.org/, human protein atlas, NCBI, ncbi.nlm.nih. gov/, ncbi.nlm.nih.gov/geo/, portal.gdc.cancer.gov/, proteinatlas. org/, The Cancer Genome Atlas

\section{AUTHOR CONTRIBUTIONS}

$\mathrm{XW}$ and TL have equally contributed to this article as first authors. BP and WC have equally contributed to this article as last and corresponding authors. Conception and design: BP, WC, XW, and TL. Administrative support: BP and WC. Provision of study materials or patients: XW and TL. Collection and assembly of data: XW, TL, ML, JL, and XW. Data analysis and interpretation: All authors. Manuscript writing: All authors. Final approval of manuscript: All authors.

\section{FUNDING}

This work was supported by the Natural Science Foundation of Guangdong Province (No.: 2018A030313529).

\section{SUPPLEMENTARY MATERIAL}

The Supplementary Material for this article can be found online at: https://www.frontiersin.org/articles/10.3389/fmolb.2021.621232/ full\#supplementary-material.

platelet ratio and fibrinogen in patients with HBV-related hepatocellular carcinoma after hepatectomy. Am. J. Tourism Res. 12 (6), 2984-2997.

De Stefano, F., Chacon, E., Turcios, L., Marti, F., and Gedaly, R. (2018). Novel biomarkers in hepatocellular carcinoma. Dig. Liver Dis. 50 (11), 1115-1123. doi:10.1016/j.dld.2018.08.019

Forner, A., Llovet, J. M., and Bruix, J. (2012). Hepatocellular carcinoma. The Lancet 379 (9822), 1245-1255. doi:10.1016/s0140-6736(11)61347-0

Fu, B., Meng, W., Zeng, X., Zhao, H., Liu, W., and Zhang, T. (2017). TXNRD1 is an unfavorable prognostic factor for patients with hepatocellular carcinoma. BioMed Res. Int. 2017, 4698167. doi:10.1155/2017/4698167

Guo, Y., Ye, Q., Deng, P., Cao, Y., He, D., Zhou, Z., et al. (2020). Spermine synthase and MYC cooperate to maintain colorectal cancer cell survival by repressing Bim expression. Nat. Commun. 11 (1), 3243. doi:10.1038/s41467-020-17067-x

Hua, S., Quan, Y., Zhan, M., Liao, H., Li, Y., and Lu, L. (2019). miR-125b-5p inhibits cell proliferation, migration, and invasion in hepatocellular carcinoma via targeting TXNRD1. Cancer Cell Int. 19, 203. doi:10.1186/s12935-019-0919-6

Huang, S., Li, J., Tam, N. L., Sun, C., Hou, Y., Hughes, B., et al. (2019a). Uridinecytidine kinase 2 upregulation predicts poor prognosis of hepatocellular carcinoma and is associated with cancer aggressiveness. Mol. Carcinog. 58 (4), 603-615. doi:10.1002/mc.22954

Huang, X., Gan, G., Wang, X., Xu, T., and Xie, W. (2019b). The HGF-MET axis coordinates liver cancer metabolism and autophagy for chemotherapeutic resistance. Autophagy 15 (7), 1258-1279. doi:10.1080/15548627.2019.1580105 
Kimhofer, T., Fye, H., Taylor-Robinson, S., Thursz, M., and Holmes, E. (2015). Proteomic and metabonomic biomarkers for hepatocellular carcinoma: a comprehensive review. Br. J. Cancer 112 (7), 1141-1156. doi:10.1038/bjc. 2015.38

Lee, D., Xu, I. M., Chiu, D. K., Leibold, J., Tse, A. P., Bao, M. H., et al. (2019a). Induction of oxidative stress through inhibition of thioredoxin reductase 1 is an effective therapeutic approach for hepatocellular carcinoma. Hepatology 69 (4), 1768-1786. doi:10.1002/hep.30467

Lee, W. H., Chen, L. C., Lee, C. J., Huang, C. C., Ho, Y. S., Yang, P. S., et al. (2019b). DNA primase polypeptide 1 (PRIM1) involves in estrogen-induced breast cancer formation through activation of the G2/M cell cycle checkpoint. Int J Cancer 144 (3), 615-630. doi:10.1002/ijc.31788

Li, W., Xiao, J., Zhou, X., Xu, M., Hu, C., Xu, X., et al. (2015). STK4 regulates TLR pathways and protects against chronic inflammation-related hepatocellular carcinoma. J. Clin. Invest. 125 (11), 4239-4254. doi:10.1172/JCI81203

Liu, G. M., Xie, W. X., Zhang, C. Y., and Xu, J. W. (2020). Identification of a fourgene metabolic signature predicting overall survival for hepatocellular carcinoma. J. Cell. Physiol. 235 (2), 1624-1636. doi:10.1002/jcp.29081

Loglio, A., Iavarone, M., Facchetti, F., Di Paolo, D., Perbellini, R., Lunghi, G., et al. (2020). The combination of PIVKA-II and AFP improves the detection accuracy for HCC in HBV caucasian cirrhotics on long-term oral therapy. Liver Int. 40 (8), 1987-1996. doi:10.1111/liv.14475

Mitchell, M. C., Friedman, L. S., and McClain, C. J. (2017). Medical management of severe alcoholic hepatitis: expert review from the clinical practice updates committee of the AGA institute. Clin. Gastroenterol. Hepatol. 15 (1), 5-12. doi:10.1016/j.cgh.2016.08.047

Nekvindova, J., Mrkvicova, A., Zubanova, V., Hyrslova Vaculova, A., Anzenbacher, P., Soucek, P., et al. (2020). Hepatocellular carcinoma: gene expression profiling and regulation of xenobiotic-metabolizing cytochromes P450. Biochem. Pharmacol. 177, 113912. doi:10.1016/j.bcp.2020.113912

Notarpaolo, A., Layese, R., Magistri, P., Gambato, M., Colledan, M., Magini, G., et al. (2017). Validation of the AFP model as a predictor of HCC recurrence in patients with viral hepatitis-related cirrhosis who had received a liver transplant for HCC. J. Hepatol. 66 (3), 552-559. doi:10.1016/j.jhep.2016. 10.038

Piccinin, E., Villani, G., and Moschetta, A. (2019). Metabolic aspects in NAFLD, NASH and hepatocellular carcinoma: the role of PGC1 coactivators. Nat. Rev. Gastroenterol. Hepatol. 16 (3), 160-174. doi:10. 1038/s41575-018-0089-3

Pinyol, R., Montal, R., Bassaganyas, L., Sia, D., Takayama, T., Chau, G. Y., et al. (2019). Molecular predictors of prevention of recurrence in HCC with sorafenib as adjuvant treatment and prognostic factors in the phase 3 STORM trial. Gut 68 (6), 1065-1075. doi:10.1136/gutjnl-2018-316408

Sangineto, M., Villani, R., Cavallone, F., Romano, A., Loizzi, D., and Serviddio, G. (2020). Lipid metabolism in development and progression of hepatocellular carcinoma. Cancers 12 (6). doi:10.3390/cancers12061419

Tang, Z., Li, C., Kang, B., Gao, G., Li, C., and Zhang, Z. (2017). GEPIA: a web server for cancer and normal gene expression profiling and interactive analyses. Nucl. Acids Res. 45 (W1), W98-W102. doi:10.1093/nar/gkx247
Tuo, L., Xiang, J., Pan, X., Gao, Q., Zhang, G., Yang, Y., et al. (2018). PCK1 downregulation promotes TXNRD1 expression and hepatoma cell growth via the nrf2/Keap1 pathway. Front. Oncol. 8, 611. doi:10.3389/fonc.2018.00611

Wang, Z., Zhu, J., Liu, Y., Liu, C., Wang, W., Chen, F., et al. (2020). Development and validation of a novel immune-related prognostic model in hepatocellular carcinoma. J. Transl. Med. 18 (1), 67. doi:10.1186/s12967-020-02255-6

$\mathrm{Xu}, \mathrm{H} ., \mathrm{Ma}, \mathrm{J} ., \mathrm{Wu}, \mathrm{J}$., Chen, L., Sun, F., Qu, C., et al. (2016). Gene expression profiling analysis of lung adenocarcinoma. Braz. J. Med. Biol. Res. 49 (3). doi:10. 1590/1414-431X20154861

Xu, Q., Yan, Y., Gu, S., Mao, K., Zhang, J., Huang, P., et al. (2018). A novel inflammation-based prognostic score: the fibrinogen/albumin ratio predicts prognoses of patients after curative resection for hepatocellular carcinoma. J. Immunol. Res. 48, 4925498. doi:10.1155/2018/4925498

Yamamoto, K., Imamura, H., Matsuyama, Y., Kume, Y., Ikeda, H., Norman, G. L., et al. (2010). AFP, AFP-L3, DCP, and GP73 as markers for monitoring treatment response and recurrence and as surrogate markers of clinicopathological variables of HCC. J. Gastroenterol. 45 (12), 1272-1282. doi:10.1007/s00535-010-0278-5

Yin, L., He, N., Chen, C., Zhang, N., Lin, Y., and Xia, Q. (2019). Identification of novel blood-based HCC-specific diagnostic biomarkers for human hepatocellular carcinoma. Artif. Cells Nanomed. Biotechnol. 47 (1), 1908-1916. doi:10.1080/21691401.2019.1613421

Zhang, B., and Finn, R. S. (2016). Personalized clinical trials in hepatocellular carcinoma based on biomarker selection. Liver Cancer 5 (3), 221-232. doi:10. $1159 / 000367763$

Zhang, C., Peng, L., Zhang, Y., Liu, Z., Li, W., Chen, S., et al. (2017). The identification of key genes and pathways in hepatocellular carcinoma by bioinformatics analysis of high-throughput data. Med. Oncol. 34 (6), 101. doi:10.1007/s12032-017-0963-9

Zhang, Y., Zhang, L., Xu, Y., Wu, X., Zhou, Y., and Mo, J. (2020). Immune-related long noncoding RNA signature for predicting survival and immune checkpoint blockade in hepatocellular carcinoma. J. Cell. Physiol. 235 (12), 9304-9316. doi: $10.1002 /$ jcp. 29730

Zhou, Q., Jiang, H., Zhang, J., Yu, W., Zhou, Z., Huang, P., et al. (2018). Uridinecytidine kinase 2 promotes metastasis of hepatocellular carcinoma cells via the Stat3 pathway. Cancer Manag. Res. 10, 6339-6355. doi:10.2147/CMAR. S182859

Conflict of Interest: The authors declare that the research was conducted in the absence of any commercial or financial relationships that could be construed as a potential conflict of interest.

Copyright (c) $2021 \mathrm{Wu}, \mathrm{Lan}, \mathrm{Li}, \mathrm{Liu}, \mathrm{Wu}$, Shen, Chen and Peng. This is an openaccess article distributed under the terms of the Creative Commons Attribution License (CC BY). The use, distribution or reproduction in other forums is permitted, provided the original author(s) and the copyright owner(s) are credited and that the original publication in this journal is cited, in accordance with accepted academic practice. No use, distribution or reproduction is permitted which does not comply with these terms. 